INPLASY

PROTOCOL

To cite: Xiao et al. Effectiveness and safety of Massage in the treatment of the congenital

muscular torticollis: a

systematic review and metaanalysis Protocol. Inplasy protocol 202070086. doi: 10.37766/inplasy2020.7.0086

Received: 19 July 2020

Published: 19 July 2020

Corresponding author:

Yuanyi Xiao

xyy0204@126.com

Author Affiliation:

The Affiliated hospital-Jiangxi University of TCM

Support: Grant number: 81860877, 816608

Review Stage at time of this submission: The review has not yet started.

Conflicts of interest: No.

\section{Effectiveness and safety of Massage in the treatment of the congenital muscular torticollis: a systematic review and meta-analysis Protocol}

Xiao, Y1; Chi, Z2; Yuan, F3; Zhu, D4; Ouyang, X5; Xu, W6; Li, J7; Luo, Z8; Chen, R9; Jiao, $\mathrm{R}^{10}$.

Review question / Objective: Massage has been widely used in the treatment of muscular torticollis in children, but there is no objective and systematic evaluation of the efficacy of various literature, and the efficacy of massage in the treatment of CMT is not clear. The purpose of this study is to evaluate the clinical efficacy and safety of massage in the treatment of muscular torticollis in children. Relevant randomized controlled trials (RCTs) will be searched from the databases of PubMed, the Cochrane Library, Embase, the China National Knowledge Infrastructure, Wanfang Database, Chinese Science and Technology Periodical Database, and Chinese Biomedical Literature Database from their inception to May 2020. Two reviewers will independently select studies, collect data, and assess the methodology quality by the Cochrane risk of bias tool. The RevMan V.5.3 will be used for meta-analysis. This study will provide an assessment of the current state of Chinese massage therapy for the congenital muscular torticollis, aiming to show the efficacy and safety of massage treatment.

INPLASY registration number: This protocol was registered with the International Platform of Registered Systematic Review and Meta-Analysis Protocols (INPLASY) on 19 July 2020 and was last updated on 19 July 2020 (registration number INPLASY202070086).

\section{INTRODUCTION}

Review question / Objective: Massage has been widely used in the treatment of muscular torticollis in children, but there is no objective and systematic evaluation of the efficacy of various literature, and the efficacy of massage in the treatment of CMT is not clear. The purpose of this study is to evaluate the clinical efficacy and safety of massage in the treatment of muscular torticollis in children. Relevant randomized controlled trials (RCTs) will be searched from the databases of PubMed, 
the Cochrane Library, Embase, the China National Knowledge Infrastructure, Wanfang Database, Chinese Science and Technology Periodical Database, and Chinese Biomedical Literature Database from their inception to May 2020. Two reviewers will independently select studies, collect data, and assess the methodology quality by the Cochrane risk of bias tool. The RevMan V.5.3 will be used for metaanalysis. This study will provide an assessment of the current state of Chinese massage therapy for the congenital muscular torticollis, aiming to show the efficacy and safety of massage treatment.

Condition being studied: Congenital muscular torticollis(CMT), also known as synonymously with congenital torticollis, is a common disorder of the musculoskeletal system in neonates and infants. CMT is defined as torticollis caused by fibrosis contracture of the sternocleidomastoid muscle on one side. Its typical clinical characteristics are that the child's head is biased toward the affected side and the face is turned to the healthy side. CMT usually occurs during the neonatal period or after birth. Epidemiological studies have shown that the incidence of CMT is about $0.3 \%$ to $2 \% 5$. In addition, recent studies have found that the incidence of CMT shows an upward trend6. CMT is the third most common congenital musculoskeletal deformity in children after congenital hip dysplasia and calcaneovalgus feet, which has a serious impact on the quality of life of patients and their families. If CMT cannot be treated in time, the deformity will gradually become apparent as we age and the growth and development of skeletal muscle. In addition, secondary sequelae related to CMT including visual dysfunction, facial asymmetry, delayed development, cervical scoliosis and vertebral wedge degeneration will have a serious impact on the child's appearance and even mental health.

\section{METHODS}

Search strategy: Search from the establishment of the database to May 1, 2020. We will search 4 English medical electronic databases including PubMed, EMBASE, Cochrane Central Register of Controlled Trials, Web of Science. Chinese literature will be searched through China's four major databases, including Chinese Biomedical Literature Database (CBM), Wanfang Database (WF), the Chongqing VIP (VIP) and Chinese National Knowledge Infrastructure (CNKI).

Participant or population: All cases included in the trial involved participants who had been diagnosed with CMT, Diagnostic criteria for CMT, according to "Schools of pediatric massage23" "tuinaology24" and"the 2018 Congenital Muscular Torticollis Clinical Practice Guideline 2" The diagnosis relies mainly on clinical and physical examination findings25. include a persistent lateral flexion of the head to the affected side and cervical rotation to the opposite side; a palpable, intramuscular, fibrotic mass (fibromatosis colli) in the affected sternocleidomastoid (SCM) muscle; secondary sequelae include plagiocephaly, facial asymmetry, and developmental delay, The affected side is smaller than the healthy side; also, exclude the Visual impairment compensated for postural torticollis, spinal deformity caused by bony torticollis, and nerve torticollis caused by cervical muscle paralysis. There is no restriction of age, sex, or race limit.

Intervention: The interventions of the experimental group will include any type of clinical massage for CMT alone to improve the symptoms. include Chinese massage, tuina, acupressure, therapeutic massage, general massage, acupressure, relaxation, etc. Massage Combined with other interventions such as acupuncture, moxibustion, herbal medicine, qigong, functional exercise and other mixed therapies will be excluded.

Comparator: The controlled intervention accepts any international recognized therapy, such as traditional medicine, acupuncture, etc. Non-intervention and Placebo will also be included. Studies comparing the therapeutic effects of 
different types of Massage manipulations will be excluded.

Study designs to be included: All the RCTs of Massage for the management of Children with CMT patients will be included without publication status restriction.

Eligibility criteria: All randomized controlled trials (RCT s) study on massage therapy treatment of Functional severe constipation.Others such as case reports, animal experiments, non-RCTs, or RCT protocol will be excluded.

Information sources: Electronic data sources. Search from the establishment of the database to May 1, 2020. We will search 4 English medical electronic databases including PubMed, EMBASE, Cochrane Central Register of Controlled Trials, Web of Science. Chinese literature will be searched through China's four major databases, including Chinese Biomedical Literature Database (CBM), Wanfang Database (WF), the Chongqing VIP (VIP) and Chinese National Knowledge Infrastructure (CNKI). The searching strategy of PubMed is presented in table. Search Other resources. We will search the Clinicaltrials.gov, China Clinical Trial Registry, and the relevant conference papers related to Massage for treatment of congenital muscular torticollis, proposing to obtain unpublished or ongoing has not uploaded trial data.

Main outcome(s): Head posture range of motion (ROM) and Neck rotation cervical ROM will be adopted as the primary outcomes. Changes of head deflection angle, active-passive cervical ROM between before treatment and follow-up, measured by the clinical examination. A study indicate that decisions guiding treatment for CMT are based on clinical examination rather than on ultrasound findings.

Additional outcome(s): 1. Use ultrasound and color Doppler imaging to observe Change of fibrotic mass in the affected sternocleidomastoid (SCM) muscle before treatment and during follow-up, such as the thickness, heterogenous echogenicity, and asymmetry of the SCM muscle, mass size. Many studies show that ultrasonography is generally accepted as the primary evaluation tool for CMT, can objectively respond to treatment effects 27. 2.Change in Clinical symptom score from baseline to the last available follow-up. The score included lateral head-righting, sternocleidomastoid tumor thickness, early motor skills, development of symmetrical movement, craniofacial asymmetry changes, parental stress, compliance with home exercises, overall improvement, and other possible outcomes potentially reflecting changes in the subject's condition. 3. The incidence rate of adverse events.

Quality assessment / Risk of bias analysis: Two reviewers (FQ and DC) of the team will use the risk of bias assessment tool by the Cochrane Collaboration to independently evaluate the quality of the final included trials28.The risk assessment indicators will include the following Seven contents: random sequence generation, allocation concealment, blinding of participants and personnel, blinding of outcome assessment, incomplete outcome data, selective reporting, and other sources of bias. Each domain of the study will be judged as high-risk, low-risk, and unclear risk of bias as the evaluation results. Our reviewers (FQ and DC) will strictly check the assessment results in accordance with the evaluation rules. If there is any ambiguity and disagreement, we will be resolved through consultation. In addition, it can also be judged by the third reviewer(XL).

Strategy of data synthesis: We will designate two reviewers ( $\mathrm{YY}$ and $\mathrm{ZH}$ ) use Review Manager Software (RevMan 5.3) and Stata software to conduct statistical analysis and synthesize all data. For categorical data, we will use the risk ratio (RR) and $95 \%$ confidence intervals(CLs) to calculate and summarize data. For continuous data, Mean difference (MD) and 95\% confidence intervals (Cls) will be used to present the data synthesis outcome. If the outcome variables of different 
measurement scales are measured, standardized mean difference analysis (SMDS) with $95 \% \mathrm{Cl}$ will be performed.

Subgroup analysis: If there are significant heterogeneities in the included studies, the STATA software will be used for subgroup analysis and meta-regression analysis according to the characteristics of the test subjects, sample size, different massage intervention methods, quality of included trials, etc.

Sensibility analysis: We will evaluate the robustness of the meta-analysis results through sensitivity analysis, and exclude such as small-sample trials and low-quality trials to explore the impact of trial quality on efficacy estimates. In addition, we will conduct a second meta-analysis based on the results of the sensitivity analysis, summarize in tables and discuss.

Country(ies) involved: China.

Keywords: Massage, congenital muscular torticollis, protocol, systematic review.

Contributions of each author:

Author 1 - Yuanyi Xiao - The author drafted the manuscript.

Author 2 - Zhenhai Chi - The author provided statistical expertise.

Author 3 - Fuqiang Yuan.

Author 4 - Daocheng Zhu.

Author 5 - Xilin Ouyang.

Author 6 - Wei Xu.

Author 7 - Jun Li.

Author 8 - Zhaona Luo.

Author 9 - Rixin Chen.

Author 10 - Lin Jiao - The author contributed to the development of the selection criteria, and the risk of bias assessment strategy. 